\title{
Do NKT cells control autoimmunity?
}

\author{
Lucienne Chatenoud \\ INSERM U25, IRNEM, Hôpital Necker, Paris, France \\ J. Clin. Invest. 110:747-748 (2002). doi:10.1172/JCI200216625.
}

Extensive work in the early 90s established the importance of the Th1/Th2 paradigm and showed that Th2 $\mathrm{CD}^{+}$ lymphocytes can dampen Th1-mediated immune responses. Later experiments highlighted the importance of a new subset of lymphocytes, termed NKT cells, in the regulation of Th2 cell differentiation. These NKT cells are distinct from other thymus-derived lymphocytes in that they express some markers typical of NK cells, as well as a quasi-invariant $\mathrm{T}$ cell receptor (TCR) type. Following stimulation of this TCR, NKT cells produce large amounts of IL-4, which drives the differentiation and growth of Th2 cells.

This finding suggested that NKT cells could directly control physiological Th2-driven immune responses and thus indirectly regulate Th1-mediated autoimmunity $(1,2)$. Because NKT cells are abundant in the liver and bone marrow but are present in very small numbers in peripheral lymphoid organs $(1,2)$, this hypothesis was initially controversial, but it soon received strong support from experiments performed in genetically manipulated mice. In particular, work with mice lacking the $\beta 2$ microglobulin chain, which are NKT cell-deficient because they lack the MHC class I-restricting element CD1d, indicated that NKT cells are indeed important players in Th2 differentiation and $\operatorname{IgE}$

\footnotetext{
Address correspondence to: Lucienne Chatenoud, INSERM U25, Hôpital Necker, 161 Rue de Sèvres, 75015 Paris, France. Phone: 33-1-44495371; Fax: 33-1-43062388; E-mail: chatenoud@necker.fr.

Conflict of interest: No conflict of interest has been declared.

Nonstandard abbreviations used: $\mathrm{T}$ cell receptor (TCR) ; nonobese diabetic (NOD); $\alpha$-galactosyl ceramide $(\alpha$-GalCer); experimental allergic encephalomyelitis (EAE).
}

production (3). Moreover, Vo14J $\alpha 281$ transgenic mice, which harbour a large population of NKT cells, show heightened Th2 polarization and high serum levels of IgE (4).

A remarkable number of experiments in various disease-prone mouse strains now link defective NKT cell function with autoimmunity. Thus, initial data from Gombert et al. $(5,6)$, subsequently confirmed by other groups $(7,8)$, showed a major deficiency of NKT cell numbers in nonobese diabetic (NOD) mice, a well-established model of spontaneous, autoimmune T-cell mediated insulin-dependent diabetes (9). Both the number of NOD NKT cells and the functional capacity of these cells, as assessed by IL-4 release following TCR ligation, are dramatically reduced $(5-8,10)$. Moreover, in vivo treatment with the specific NKT cell TCR ligand $\alpha$-galactosyl ceramide ( $\alpha$-GalCer, a glycolipid derived from a marine sponge) prevents disease in NOD mice $(10,11)$. Over-expression of NKT cell-associated Vo14Jo281 TCR chain in transgenic mice has a similar effect (12). Conversely, the onset of diabetes was recently reported to be accelerated in NOD mice lacking CD1d expression $(13,14)$, again consistent with a pathogenic role for NKT cell deficiency.

Work in another model of autoimmunity, experimental allergic encephalomyelitis (EAE), also provides evidence that NKT cells help control this pathogenic process. Mice of the SJL strain, which exhibit a unique tendency to develop chronic EAE, show the same numerical and functional NKT cell deficiency as NOD mice (15). NKT cell activation using $\alpha$-GalCer protects mice from EAE (16-18). NOD mice harboring the V $\alpha 14 J \alpha 281$ TCR transgene are protected from EAE (19), just as they are for diabetes
(12). This compelling evidence from the two best established animals models of autoimmunity provided the rationale for investigating the role of NKT cells in human autoimmune disease. Unfortunately, such studies in human patients are intrinsically limited by the poor representation of NKT cells among circulating mononuclear cells.

In their report in this issue of the JCI, Albert Bendelac's group has characterized NKT cells using double staining with a CD1d- $\alpha$-GalCer tetramer and a Vo24 specific antibody. They find that the numbers of NKT cells do not differ between diabetic patients, subjects at high risk for developing the disease, and normal controls (20). IL-4 production by NKT cells was also similar among these groups, as assessed by intracytoplasmic staining following short-term PMA and ionomycin stimulation (20). These observations are in clear distinction with previous reports showing both a numerical and a functional defect in the IL-4 producing ability of NKT cells in diabetic patients, as compared to both discordant twins and normal controls $(21,22)$. At this stage, it is hard to interpret the discrepancies between these results, since the methods for detecting NKT cells and the functional assays employed differ significantly between these studies. While the use of CD1d tetramers in the present report (20) adds considerably to the specificity of detection, this protocol may also identify low avidity tetramer-binding cells whose biological relevance is questionable. Moreover, the assumption that $\alpha$-GalCer binds to all NKT cells may be incorrect in light of some reports showing that there are invariant and non-invariant CD1d-restricted $\mathrm{T}$ cells that do not respond to this 
glycolipid $(23,24)$. Hence, the doublestaining procedure applied here may have missed some NKT cells. Conversely, since $\alpha$-GalCer loaded CD1d tetramers have been reported to stain CD1d-restricted $T$ cells not expressing the invariant Vo24 TCR chain (23, 25 ), it is also possible that some of the cells identified by this procedure are not NKT cells. Finally, because Bendelac's group (20) examined whole mononuclear cells that had been triggered with PMA and ionomycin, it is not possible to compare the present functional data with those of earlier reports, where clones of NKT cells were expanded in culture and stimulated in a more physiological manner via the TCR (21).

Thus, the role of NKT cells in autoimmunity is still unsettled and will only be resolved by the consistent use of techniques and reagents. If NKT cells are found to play a role in controlling human autoimmunity, as they appear to do in mouse models, this effect must be understood in the context of the other well documented regulatory $\mathrm{T}$ cells, notably $\mathrm{Th} 2$ and $\mathrm{CD} 25^{+} \mathrm{T}$ cells (26). Whether NKT cells interact with these subsets to promote their differentiation or survival is a question of great interest.

1. Bendelac, A., Rivera, M.N., Park, S.H., and Roark, J.H. 1997. Mouse CD1-specific NK1 T cells: development, specificity and function. Annu. Rev. Immunol. 15:535-562.
2. Porcelli, S.A., and Modlin, R.L. 1999. The CD1 system: antigen-presenting molecules for $\mathrm{T}$ cell recognition of lipids and glycolipids. Annu. Rev. Immunol. 17:297-329.

3. Yoshimoto, T., Bendelac, A., Watson, C., Hu-Li, J., and Paul, W.E. 1995. Role of NK1.1+ T cells in a $\mathrm{TH} 2$ response and in immunoglobulin $\mathrm{E}$ production. Science. 270:1845-1847.

4. Bendelac, A., Hunziker, R.D., and Lantz, O. 1996. Increased interleukin 4 and immunoglobulin $\mathrm{E}$ production in transgenic mice overexpressing NK1 T cells. J. Exp. Med. 184:1285-1293.

5. Gombert, J.M., et al. 1996. Early quantitative and functional deficiency of NK1(+)- like thymocytes in the NOD mouse. Eur. J. Immunol. 26:2989-2998.

6. Gombert, J.M., et al. 1996. IL-7 reverses NK1+ T cell-defective IL-4 production in the non-obese diabetic mouse. Int. Immunol. 8:1751.

7. Baxter, A.G., Kinder, S.J., Hammond, K.J.L., Scollay, R., and Godfrey, D.I. 1997. Association between $\alpha \beta T C R+C D 4-C D 8-$ T-cell deficiency and IDDM in NOD/Lt mice. Diabetes. 146:572-582.

8. Hammond, K.I.L., et al. 1998. alpha/beta-T cell receptor (TCR)+CD4-CD8- (NKT) thymocytes prevent insulin-dependent diabetes mellitus in nonobese diabetic (NOD)/Lt mice by the influence of interleukin (IL)-4 and/or IL-10. J. Exp Med. 187:1047.

9. Bach, JF. 1994. Insulin-dependent diabetes mellitus as an autoimmune disease. Endocrine Rev 15:516-542.

10. Sharif, S., et al. 2001. Activation of natural killer $\mathrm{T}$ cells by alpha-galactosylceramide treatment prevents the onset and recurrence of autoimmune Type 1 diabetes. Nat. Med. 7:1057-1062.

11. Hong, S., et al. 2001. The natural killer T-cell ligand alpha-galactosylceramide prevents autoimmune diabetes in non-obese diabetic mice. Nat. Med. 7:1052-1056

12. Lehuen, A., et al. 1998. Overexpression of natural killer T cells protects $\mathrm{V}$ alpha 14-J alpha 281 transgenic nonobese diabetic mice against diabetes. J. Exp. Med. 188:1831-1839.

13. Wang, B., Geng, Y.B., and Wang, C.R. 2001. Cd1restricted NK t cells protect nonobese diabetic mice from developing diabetes. J. Exp. Med. 194:313-320.
14. Shi, F.D., et al. 2001. Germ line deletion of the CD1 locus exacerbates diabetes in the NOD mouse. Proc. Natl. Acad. Sci. USA. 98:6777-6782.

15. Yoshimoto, T., Bendelac, A., Hu-Li, J., and Paul, W.E. 1995. Defective IgE production by SJL mice is linked to the absence of CD4+, NK1.1+ T cells that promptly produce interleukin 4. Proc. Natl. Acad. Sci. USA. 92:11931-11934.

16. Singh, A.K., et al. 2001. Natural killer T cell activation protects mice against experimental autoimmune encephalomyelitis. J. Exp. Med. 194:1801-1811.

17. Jahng, A.W., et al. 2001. Activation of natural killer $\mathrm{T}$ cells potentiates or prevents experimental autoimmune encephalomyelitis. J. Exp. Med. 194:1789-1799.

18. Miyamoto, K., Miyake, S., and Yamamura, T 2001. A synthetic glycolipid prevents autoimmune encephalomyelitis by inducing TH2 bias of natural killer T cells. Nature. 413:531-536

19. Mars, L.T., et al. 2002. Cutting edge: V alpha 14-J alpha 281 NKT cells naturally regulate experimental autoimmune encephalomyelitis in nonobese diabetic mice. J. Immunol. 168:6007-6013.

20. Lee, P.T., et al. 2002. Testing the NKT cell hypothesis of human IDDM pathogenesis. J. Clin. Invest. 110:793-800. doi:10.1172/JCI200215832.

21. Wilson, S.B., et al. 1998. Extreme Th1 bias of invariant Va24JaQ $\mathrm{T}$ cells in type 1 diabetes. Nature. 391:177-181.

22. Kukreja, A., et al. 2002. Multiple immuno-regulatory defects in type-1 diabetes. J. Clin. Invest. 109:131-140. doi:10.1172/JCI200213605.

23. Gumperz, J.E., et al. 2000. Murine CD1d-restricted $\mathrm{T}$ cell recognition of cellular lipids. Immunity. 12:211-221.

24. Behar, S.M., Podrebarac, T.A., Roy, C.J., Wang, C.R., and Brenner, M.B. 1999. Diverse TCRs recognize murine CD1. J. Immunol. 162:161-172.

25. Gadola, S.D., Dulphy, N., Salio, M., and Cerundolo, V. 2002. Valpha24-JalphaQ-independent, CD1d-restricted recognition of alpha-galactosylceramide by human CD4(+) and CD8alphabeta(+) T lymphocytes. J. Immunol. 168:5514.

26. Bach, J.F., and Chatenoud, L. 2001. Tolerance to islet autoantigens in type 1 diabetes. Annu. Rev. Immunol. 19:131-161. 